\title{
USO DE EQUIPAMENTO DE PROTEÇÃO INDIVIDUAL EM UM SERVIÇO DE ATENÇÃO DOMICILIAR
}

\author{
Jéssica Fernanda Corrêa Cordeiro ${ }^{1}$, Amanda Pavinski Alves ${ }^{2}$, Elucir Gir ${ }^{3}$, Diego Oliveira Miranda ${ }^{4}$, Silvia Rita \\ Marin da Silva Canini ${ }^{3}$
}

\begin{abstract}
RESUMO: Trata-se de um estudo transversal conduzido num Serviço de Atenção Domiciliar do interior paulista. Teve como objetivos identificar o uso de Equipamento de Proteção Individual, dispositivos de segurança, descarte de materiais perfurocortantes e fatores que dificultam e/ou facilitam o seu uso por profissionais da equipe de enfermagem. A população foi composta por 45 participantes e todos relataram usar Equipamento de Proteção Individual, sendo que $100 \%$ referiram usar luvas e os procedimentos frequentes para o uso foram curativos e administração de medicamentos. Apesar de relatarem que não há dificuldade para utilizar os Equipamentos de Proteção Individual e que se preocupam com sua própria segurança, observou-se que a adesão não foi integral. Assim, são necessários estudos futuros capazes de avaliar como ocorre de fato a assistência à saúde dos usuários nos domicílios, para que estratégias de prevenção possam ser identificadas e incorporadas à prática desses profissionais.
\end{abstract}

DESCRITORES: Equipe de enfermagem; Equipamento de proteção individual; Exposição à agentes biológicos; Serviços de assistência domiciliar.

\section{USE OF PERSONAL PROTECTIVE EQUIPMENT IN A HOME CARE SERVICE}

\begin{abstract}
A cross-sectional study was conducted at a home care service in the state of São Paulo. The objective of the study was to identify the use of personal protective equipment, safety devices, the disposal of sharp objects, and other factors that make difficult and/or facilitate such use by professionals of the nursing team. The population was made up of 45 participants who reported using personal protective equipment. They all reported using gloves, frequent procedures that required their use were dressings and administration of medications. Although it was reported that the participants did not have difficulty in using personal protective equipment, and that they were concerned about their own safety, full adherence was lacking. Therefore, further studies that are able to evaluate how home care services really occur are necessary, so that prevention strategies can be identified and incorporated into professionals' practice.

DESCRIPTORS: Nursing team; Personal Protective Equipment; Exposure to Biological Agents; Home Care Services.
\end{abstract}

\section{USO DE EQUIPOS DE PROTECCIÓN PERSONAL EN SERVICIO DE ATENCIÓN DOMICILIARIA}

RESUMEN: Estudio transversal realizado en un Servicio de Atención Domiciliaria del interior paulista, con el objetivo de identificar el uso de Equipos de Protección Personal, mecanismos de seguridad, descarte de materiales punzocortantes y factores que dificultan y/o facilitan su utilización en profesionales del equipo de enfermería. La población se compuso de 45 participantes, todos informaron usar Equipos de Protección Personal, el 100\% afirmó usar guantes y los procedimientos para su uso frecuente fueron vendajes y administración de medicamentos. A pesar de informarse que no existe dificultado para utilizar Equipos de Protección Personal y que se preocupan por la propia seguridad, se observó que la adhesión no fue integral. Consecuentemente, existe necesidad de estudios a futuro, capaces de evaluar cómo transcurre de hecho la atención de la salud de usuarios en sus domicilios, para poder identificar e incorporar estrategias preventivas a la práctica de estos profesionales.

DESCRIPTORES: Grupo de Enfermería; Equipo de Protección Personal; Exposición a Agentes Biológicos; Servicios de Atención de Salud a Domicilio.

${ }^{1}$ Enfermeira. Mestranda em Enfermagem. Escola de Enfermagem de Ribeirão Preto da Universidade de São Paulo. Ribeirão Preto, SP, Brasil.

${ }^{2}$ Enfermeira. Mestre em Ciência. Escola de Enfermagem de Ribeirão Preto da Universidade de São Paulo. Ribeirão Preto, SP, Brasil.

${ }^{3}$ Enfermeira. Docente da Escola de Enfermagem de Ribeirão Preto da Universidade de São Paulo. Ribeirão Preto, SP, Brasil. ${ }^{4}$ Enfermeiro. Pós Doutorando da Escola de Enfermagem de Ribeirão Preto da Universidade de São Paulo. Ribeirão Preto, SP, Brasil.

Autor Correspondente:

Jéssica Fernanda Corrêa Cordeiro

Universidade de São Paulo

Av. dos Bandeirantes, 3900 - 14040-902 - Ribeirão Preto, SP, Brasil

E-mail: jessica.cordeiro@usp.br
Recebido: 27/02/2016

Finalizado: 19/09/2016 


\section{INTRODUÇÃO}

Os profissionais da área da saúde estão frequentemente expostos a acidentes ocupacionais com materiais biológicos por meio de lesões com objetos pontiagudos, como agulhas contaminadas com sangue, espirros de secreção em mucosas ou pele não íntegra ${ }^{(1)}$. Os dados epidemiológicos dos Estados Unidos, referentes à soroconversão de profissionais da área da saúde, no período de 1985 a 2013, evidenciaram que os profissionais de enfermagem foram os que sustentaram a maior taxa de soroconversão ao Human Immunodeficiency Virus (HIV) ${ }^{(2)}$.

O risco estimado de transmissão do HIV após acidentes com materiais perfurocortantes e pacientesfonte com sorologia positiva é de 0,3 a 0,5\%, e, após exposição de membrana mucosa, é de 0,09\%. A transmissão relacionada à exposição de pele já foi documentada, porém seu risco ainda não foi quantificado. Além do HIV outros vírus como os das hepatites B e C também podem ser transmitidos após exposição ocupacional a material biológico ${ }^{(3)}$.

No Brasil, a Norma Regulamentadora (NR) nº 32 do Ministério do Trabalho, de 16 de novembro de 2005, estabelece diretrizes básicas para a implementação de medidas de proteção à saúde dos trabalhadores dos serviços de saúde, bem como daqueles que exercem atividades de promoção e assistência à saúde em geral. Essa norma tem como objetivo reduzir o número de acidentes de trabalho com material biológico, determinando que os empregadores informem seus empregados quanto aos riscos a que estão expostos. Os empregadores devem, ainda, garantir que todos os trabalhadores dos serviços de saúde tenham direito, gratuitamente, a um programa de imunização contra doenças transmissíveis; capacitação continuada e disponibilidade de Equipamento de Proteção Individual (EPI $)^{(4)}$.

A maioria dos estudos sobre acidentes ocupacionais com material biológico, potencialmente contaminado, vem sendo conduzida em instituições hospitalares ${ }^{(5)}$. Contudo, nos últimos anos, houve incremento de pesquisas com a equipe de atendimento pré-hospitalar como é o caso do atendimento domiciliar, porém, esses estudos ainda são escassos, principalmente no Brasil ${ }^{(6)}$.

Segundo a Portaria n ${ }^{\circ} 2.029$ do Ministério da Saúde de 24 de agosto de 2011, considera-se Serviço de Atenção Domiciliar (SAD) um serviço substitutivo ou complementar à internação hospitalar ou ao atendimento ambulatorial, responsável pelo gerenciamento e operacionalização das equipes Multiprofissionais de Atenção Domiciliar (EMAD) e das Equipes Multiprofissionais de Apoio (EMAP) ${ }^{(7)}$.

Por se tratar de uma política de saúde relativamente nova e um novo campo de atuação para os profissionais de enfermagem, pouco se conhece sobre as condições de trabalho em SAD, como ocorre a prática clínica e o uso de EPI. Assim, julgou-se pertinente a condução do presente estudo que teve como objetivos identificar o uso de EPI (luvas, óculos, avental e máscara), de dispositivos de segurança (agulhas retráteis, lancetas seguras) e o descarte de materiais perfurocortantes relatados por profissionais da equipe de enfermagem, durante as visitas domiciliares, e também os fatores que facilitam e/ou dificultam o uso de EPI.

\section{MÉTODO}

Trata-se de um estudo de corte transversal, conduzido com profissionais que atuavam no SAD da Secretaria Municipal da Saúde de Ribeirão Preto (SMS-RP) no período do estudo. A população elegível do estudo foi de 50 profissionais de enfermagem, mas em decorrência de cinco profissionais recusarem em participar, a população foi composta por 45 participantes. Foi incluído o profissional de enfermagem em exercício ativo e realizar procedimentos de enfermagem no domicílio. Foram excluídos os profissionais que realizavam visitas domiciliares de forma esporádica.

A coleta de dados ocorreu no período de novembro de 2014 a outubro de 2015, período em que todos os profissionais elegíveis foram abordados e convidados a participar do estudo. Ressalta-se que devido ao tipo de atividade dos profissionais, houve necessidade de o pesquisador retornar mais de uma vez à unidade para abordá-los.

Foi utilizado um roteiro de entrevista estruturado previamente submetido à apreciação de sete especialistas na temática (pré-teste), aos quais foi solicitado avaliar a clareza e a pertinência das questões 
e foi considerado adequado para o alcance dos objetivos propostos. Após coletados, realizou-se a dupla digitação dos dados em planilha do Excel e depois de corrigidos os erros de digitação, a planilha foi transportada para o programa Statistical Package for the Social Science (SPSS), versão 17.0, onde foi formatado o banco definitivo e realizadas as operações de gerenciamento das variáveis e a análise foi feita por meio de estatística descritiva.

Houve autorização do SAD e da SMS e aprovação do Comitê de Ética e Pesquisa da Escola de Enfermagem de Ribeirão Preto, sob o número 076/2014. As entrevistas foram agendadas e realizadas no próprio local de trabalho, após o término da jornada de trabalho, no período da tarde, e em sala privativa, com anuência da chefia.

\section{RESULTADOS}

A distribuição dos participantes segundo as variáveis do estudo está apresentada na Tabela 1. Observou-se que dos 45 entrevistados, 36 (80\%) era do sexo feminino, 23 (51,1\%) possuía ensino médio, 23 (51,1\%) eram auxiliares de enfermagem e 17 (37,8\%) enfermeiros. Ressalta-se que 36 (80\%) participantes possuíam um único emprego.

Observa-se na Tabela 2 que a média de idade dos participantes foi de 47,8 anos, o mínimo de tempo de trabalho no SAD foi de três meses, a média do tempo de experiência na enfermagem foi de 23,3 anos, o máximo de jornada no SAD foi de 40 horas semanais e de jornada total foi de 70 horas semanais.

Os profissionais de enfermagem que atuam no SAD foram questionados a respeito de quais procedimentos eles utilizam EPI, durante sua prática profissional no domicílio, podendo relatar mais de um procedimento. Observou-se que o uso de EPI foi mais frequentemente relatado durante a realização de curativos 36 (80\%), e nos procedimentos considerados de alto risco, como punções venosas e aspiração de vias aéreas, houve menor adesão ao uso dos EPI (Tabela 3).

Verificou-se que todos os profissionais entrevistados relataram usar EPI, porém apenas as luvas foram citadas por 45 (100\%) deles e o menos utilizado segundo o relato dos profissionais e foi o óculos de proteção 15 (33,3\%) (Tabela 4$)$.

Tabela 1 - Caracterização dos profissionais de enfermagem $(n=45)$ de um Serviço de Assistência Domiciliar, de acordo com variáveis do estudo. Ribeirão Preto, SP, Brasil, 2015

\begin{tabular}{lcc} 
Variáveis & $\mathbf{n}$ & $\mathbf{\%}$ \\
\hline Sexo & & \\
\hline Feminino & 36 & 80 \\
\hline Masculino & 9 & 20 \\
\hline Escolaridade & \\
\hline Médio & 23 & 51,1 \\
\hline Superior & 19 & 42,3 \\
\hline Especialização & 2 & 4,4 \\
\hline Mestrado & 1 & 2,2 \\
\hline Função & & \\
\hline Enfermeiro & 17 & 37,8 \\
\hline Técnico de enfermagem & 5 & 11,1 \\
\hline Auxiliar de enfermagem & 23 & 51,1 \\
\hline Número de empregos & & \\
\hline 1 & 36 & 80 \\
\hline$>1$ & 9 & 20
\end{tabular}

Tabela 2 - Caracterização dos profissionais de enfermagem $(n=45)$ de um Serviço de Assistência Domiciliar, de acordo com variáveis do estudo. Ribeirão Preto, SP, Brasil, 2015

\begin{tabular}{lcccc} 
Variávies & Média & Mínimo & Máximo & *DP \\
\hline Idade (anos) & 47,8 & 22 & 65 & 11,1 \\
\hline $\begin{array}{l}\text { Tempo de } \\
\text { experiência no } \\
\text { SAD (meses) }\end{array}$ & 73,4 & 3 & 276 & 64,9 \\
\hline $\begin{array}{l}\text { Tempo de } \\
\text { experiência na } \\
\text { enfermagem } \\
\text { (anos) }\end{array}$ & 23,3 & 1 & 40 & 9,9 \\
\hline $\begin{array}{l}\text { Jornada de } \\
\text { trabalho semanal } \\
\text { SAD (horas) }\end{array}$ & 34,1 & 30 & 40 & 4,9 \\
\hline $\begin{array}{l}\text { Jornada de } \\
\text { trabalho semanal } \\
\text { total (horas) }\end{array}$ & 36,1 & 20 & 70 & 9,8 \\
\hline * Desvio Padrão & & & & \\
\hline
\end{tabular}


Tabela 3-Distribuição dos profissionais de enfermagem $(n=45)$ de um Serviço de Assistência Domiciliar, segundo os procedimentos que os profissionais de enfermagem utilizam Equipamento de Proteção Individual. Ribeirão Preto, SP, Brasil, 2015

\begin{tabular}{lcc} 
Variáveis & $\mathbf{n}$ & $\%$ \\
\hline Curativos & & \\
\hline Sim & 36 & 80 \\
\hline Não & 9 & 20 \\
\hline Coleta de Sangue & & \\
\hline Sim & 26 & 57,8 \\
\hline Não & 19 & 42,2 \\
\hline Aplicação de medicamentos & & \\
\hline Sim & 21 & 46,7 \\
\hline Não & 24 & 53,3 \\
\hline Troca de Traqueostomia & & \\
\hline Sim & 20 & 44,4 \\
\hline Não & 25 & 55,6 \\
\hline Punções venosas & & \\
\hline Sim & 19 & 42,2 \\
\hline Não & 26 & 57,8 \\
\hline Aspiração de Vias Aéreas & & \\
\hline Sim & 16 & 35,6 \\
\hline Não & 29 & 64,4 \\
\hline Exame físico & 13 & \\
\hline Sim & 32 & 71,1 \\
\hline Não & & \\
\hline
\end{tabular}

Tabela 4 - Distribuição dos profissionais de enfermagem $(n=45)$ de um Serviço de Assistência Domiciliar, segundo o tipo de Equipamento de Proteção Individual utilizado na prática profissional. Ribeirão Preto, SP, Brasil, 2015

\begin{tabular}{lcc} 
Variáveis & $\mathbf{n}$ & $\mathbf{\%}$ \\
\hline Uso de EPI na prática profissional & & \\
\hline Sim & 45 & 100 \\
\hline Luvas & & \\
\hline Sim & 45 & 100 \\
\hline Máscara & & \\
\hline Sim & 32 & 71,1 \\
\hline Não & 13 & 28,9 \\
\hline Avental & & \\
\hline Sim & 24 & 53,3 \\
\hline Não & 21 & 46,7 \\
\hline Óculos & \\
\hline Sim & 15 & 33,3 \\
\hline Não & 30 & 66,7
\end{tabular}

Na Tabela 5, observa-se a distribuição das variáveis segundo as condições e relatos da prática na Assistência Domiciliar. $\mathrm{N}=33(73,3 \%)$ profissionais relataram que o serviço fornece dispositivos de segurança e que fazem uso desses dispositivos, $\mathrm{n}=40(88,9 \%)$ referiram levar para o domicílio o descartador de material perfurocortante. Em relação aos fatores que facilitam o uso de EPI, n=19 (42,2\%) profissionais relataram que a preocupação com sua segurança é um facilitador e $n=25(55,6 \%)$ alegaram não existir dificuldades para seu uso.

\section{- DISCUSSÃO}

Dentre os 45 indivíduos entrevistados pertencentes à equipe de enfermagem do SAD, houve predominância de mulheres. Resultados semelhantes podem ser evidenciados em outros estudos ${ }^{(8)}$. Segundo dados do Conselho Federal de Enfermagem (COFEN), 84,6\% do contingente da equipe de enfermagem, no Brasil, é formado por mulheres ${ }^{(9)}$. Contudo, atualmente observa-se um aumento significativo do sexo masculino na enfermagem.

Observou-se que a maioria dos profissionais possuía ensino médio e era auxiliar de enfermagem, dados que são corroborados por outros pesquisadores ${ }^{(10)}$. Ressalta-se que $20 \%$ dos sujeitos relataram ter mais de um emprego. O trabalho de enfermagem requer da equipe convivência com sofrimento, turnos ininterruptos, más condições de trabalho, muita responsabilidade e pouca valorização, tendo mostrado a insatisfação, o adoecimento e o aumento da evasão desses profissionais ${ }^{(11)}$.

A duplicidade de emprego, necessária à sobrevivência nos dias atuais em virtude da redução do poder aquisitivo da população, desgasta a condição física e psíquica dos profissionais. A necessidade de mais um emprego exige da equipe de enfermagem a permanência da maioria dos seus anos 
Tabela 5 - Distribuição dos profissionais de enfermagem $(n=45)$ de um Serviço de Assistência Domiciliar, segundo as condições e relatos da prática na Assistência Domiciliar. Ribeirão Preto, SP, Brasil, 2015

\begin{tabular}{lcc}
\hline Variáveis & $\mathbf{n}$ & $\%$ \\
\hline O Serviço fornece dispositivo de segurança & 33 & 73,3 \\
\hline Sim & 1 & 2,2 \\
\hline Não & 11 & 24,5 \\
\hline Às vezes & 33 & 73,3 \\
\hline Utiliza dispositivo de segurança & 1 & 2,2 \\
\hline Sim & 11 & 24,5 \\
\hline Não & & \\
\hline Quando possui & 40 & 88,9 \\
\hline Local de descarte de perfurocortante & 5 & 11,1 \\
\hline Descarta em coletor pequeno & & \\
\hline Recolhe em recipientes improvisados e descarta na Unidade & 13 & 28,9 \\
\hline Fatores que facilitam o uso de EPI & 5 & 11,1 \\
\hline Ter os EPI disponíveis & 19 & 42,2 \\
\hline Não conhecer o paciente & 8 & 17,8 \\
\hline Preocupação com a sua segurança & & \\
\hline Consciência da prevenção & 9 & 20 \\
\hline Fatores que dificultam o uso de EPI & 3 & 6,7 \\
\hline O Serviço não fornecer os EPI & 3 & 6,7 \\
\hline Calor & 5 & 11 \\
\hline Ter que transportá-los até o domicílio & 25 & 55,6 \\
\hline Prejudica a palpação das veias nas punções & 3 & \\
\hline Nenhum & & \\
\hline
\end{tabular}

produtivos em ambiente assistencial, aumentando o tempo de exposição aos riscos ocupacionais. Pesquisa conduzida num hospital da Alemanha identificou que os principais motivos que levaram os profissionais da área da saúde a se acidentarem com material biológico foram as condições de trabalho estressantes, falta de EPI e o cansaço com rotinas de trabalho ${ }^{(12)}$.

Dados apresentados pelo COFEN mostram o cenário de desemprego para a categoria, sendo que $8,8 \%$ dos profissionais entrevistados relataram tal situações nos últimos 12 meses $^{(9)}$.

Pesquisas recentes realizadas em instituições hospitalares evidenciaram que os profissionais possuíam média de idade de 36,4 anos $^{(8,13)}$. Contudo, a média de idade dos sujeitos no presente estudo foi de 47,8 anos, caracterizando um grupo mais experiente.

A experiência profissional aliada à utilização correta dos EPI pode minimizar a exposição aos riscos biológicos. Por outro lado, estudo evidenciou que o despreparo profissional expresso pelo desconhecimento do uso correto das precauções-padrão e pela falta de habilidade manual e psicomotora para realizar os diversos procedimentos de enfermagem facilitaram a ocorrência de acidentes com materiais perfurocortantes ${ }^{(10)}$. No entanto, a confiança de saber realizar procedimento muitas vezes gera uma falsa sensação de segurança, contribuindo para que o profissional deixe de utilizar o equipamento adequado.

No presente estudo, os profissionais apresentaram elevada experiência na enfermagem, aproximadamente 23 anos, porém no serviço de atendimento domiciliar, a experiência foi de apenas seis anos. Embora com maior tempo de experiência na profissão, houve menor tempo no cenário de assistência domiciliar, de tal modo que há necessidade de saber mais sobre a assistência à saúde e o uso de EPI, numa realidade diferente das instituições hospitalares.

Apesar de a recomendação de utilizar os EPI ser feita com base no tipo de procedimento, sem levar em consideração o local onde a assistência é prestada, vale destacar que algumas situações fora de um http://revistas.ufpr.br/cogitare/ 
ambiente devidamente projetado para essa finalidade podem dificultar o seu uso. O domicílio tem características muito diferentes das instituições de saúde, mostrando a necessidade de se conduzir futuras investigações para explorar as condições em que ocorre o trabalho destes profissionais de saúde.

Um dos diferenciais das equipes de atenção domiciliar com as demais equipes de saúde da rede de atenção é a relação mais estreita com os usuários em cuidados no domicílio. Dessa forma, a potencialidade inovadora da atenção domiciliar se dá pela maior permeabilidade das equipes nos diferentes aspectos vivenciados pelos usuários e suas famílias, bem como a produção de cuidado ampliado, que não se restringe aos aspectos biológicos da doença, uma vez que o profissional estreita a relação com o usuário(14). Muitas vezes isso pode conferir certa segurança ao profissional, que pela proximidade do paciente pode julgar que o mesmo não é portador de nenhum micro-organismo ou doença transmissível.

Em relação aos procedimentos realizados nos domicílios, $80 \%$ dos profissionais relataram usar EPI durante a realização de curativos. A realização de curativos pode oferecer risco de exposição a sangue e outros fluídos por meio de respingos em olhos e boca. Além disso, especificamente em curativos de úlcera por pressão, é frequente o uso de bisturi para remoção de tecido desvitalizado, associando então ao procedimento o risco de acidente cortante, tanto pelo manuseio quanto pelo descarte ${ }^{(15)}$.

Porém, cabe ressaltar que em procedimentos considerados de alto risco, como administração de medicamentos por via endovenosa e punção venosa, menos da metade dos profissionais utilizava EPI, ou seja, $46,7 \%$ e $42,2 \%$ respectivamente.

No presente estudo, todos os profissionais entrevistados relataram usar luvas de procedimento, entretanto, apenas 33\% relataram o uso de óculos de proteção. Pesquisa sobre a adesão dos profissionais de enfermagem ao uso dos EPI mostrou resultados semelhantes, sendo as luvas de procedimento os EPI mais utilizados e baixa adesão de proteção às mucosas faciais, especialmente os óculos de proteção $^{(16)}$.

Pesquisadores conduziram estudo em duas Unidades Básicas de Saúde em Goiânia e encontraram que os profissionais da área da saúde não utilizaram todos os EPI recomendados aos procedimentos por órgãos normativos nacionais, comprometendo não só a sua própria segurança como a segurança dos usuários ${ }^{(16)}$.

Independentemente da quantidade de EPI disponível, observou-se no presente estudo que o seu uso foi negligenciado pelos profissionais de enfermagem. Em um estudo qualitativo que teve como objetivo investigar concepções e práticas de técnicos de enfermagem acerca da biossegurança, constatou-se que, apesar de os EPI estarem à disposição, um número significativo admitiu não utilizálos quando necessário. De acordo com a maioria dos entrevistados, a autoconfiança, o descuido e a pressa foram os fatores que contribuíram para a omissão/negligência da equipe no uso dos $\mathrm{EPI}^{(17)}$.

A maioria dos profissionais do presente estudo relatou que o serviço fornece os dispositivos de segurança e que eles os utilizam em sua prática. Além disso, os profissionais alegaram não existir dificuldades para o uso de EPI. Embora a maioria tenha citado que não existiam fatores que dificultavam a utilização de EPI, alguns alegaram a falta do material. Estudos apontam que a disponibilização dos EPI no ambiente de trabalho pode ter influência sobre a adesão dos trabalhadores aos mesmos ${ }^{(16)}$. De acordo com a NR-32, os EPI deverão estar à disposição do trabalhador nos postos de trabalho em número suficiente ${ }^{(4)}$.

O calor foi citado como um fator dificultador na adesão ao uso de EPI pelos entrevistados, mostrando que a estrutura física com ventilação e iluminação inadequadas torna seu uso incômodo, contribuindo assim para a baixa adesão. Soma-se a isso o fato de se estar em um país tropical, com temperaturas altas em condições ambientais. Dados de estudo apontaram o calor e o incômodo como fatores que dificultam o uso da máscara e do avental ${ }^{(18)}$.

Um fator que pode conferir maior segurança aos profissionais que atuam nos domicílios é a utilização de dispositivos de segurança (lancetas, agulhas retráteis, entre outros) como recomendados pela NR-32 ${ }^{(4)}$. Estudo conduzido com enfermeiros que atuavam na assistência domiciliar mostrou que não utilizavam tais dispositivos devido à dificuldade ao acesso e também por não serem fornecidos 
por suas agências ${ }^{(19)}$. Porém, o fornecimento do EPI e dos dispositivos de segurança não é garantia de que os profissionais tenham adesão ao seu uso ou os utilizem conforme recomendado.

Além da disponibilidade dos EPI, da adesão ao uso por parte do trabalhador, os equipamentos devem ser utilizados de forma correta. Estudo ${ }^{(20)}$ que avaliou o uso de EPI por profissionais da área da saúde, por meio de filmagem, encontrou 1.797 casos de comportamentos incorretos, os quais foram corrigidos. A taxa de erro permaneceu baixa nas duas primeiras semanas após a intervenção declinando após esse período, levando os autores a concluírem que a supervisão dos profissionais e o feedback sobre a utilização correta dos EPI são etapas importante e que podem contribuir para que os indivíduos adotem comportamentos de segurança padronizados.

\section{CONCLUSÃO}

Conclui-se que todos os profissionais relataram usar EPI, porém somente as luvas de procedimento foram utilizadas por $100 \%$ dos entrevistados. Destacou a realização de curativos com uso de EPI. A maioria dos profissionais relatou que o SAD oferece, além dos EPI, os dispositivos de segurança e que o fator que mais facilita o seu uso foi preocupar-se com sua própria segurança.

Apesar de a maioria relatar que não há fatores dificultadores para o uso de EPI, alguns alegaram o calor, o transporte dos materiais até o domicílio e a não disponibilização dos mesmos pelo empregador. Deste modo, estudos futuros que avaliem a prática profissional no domicílio podem contribuir para a compreensão da utilização dos EPI, num contexto diferente das instituições de saúde.

A implementação de estratégias para promover o aumento da segurança dos profissionais depende do conhecimento das situações que representam risco e dos aspectos relacionados aos próprios indivíduos e às instituições. Futuras pesquisas devem ser conduzidas no cenário da assistência domiciliar para que se conheça como realmente ocorre a prática clínica nesse cenário e que estratégias de promoção da segurança no trabalho possam ser propostas e implementadas.

Por se tratar de entrevistas, pode ser que alguns profissionais relatassem apenas o uso de EPI nas situações mais relevantes de sua prática profissional e também pode ter ocorrido o viés de recordação, uma vez que se perguntou sobre o uso de EPI durante toda a sua prática no SAD. Porém, apesar dessas limitações, o estudo permitiu identificar as situações de uso de EPI que podem direcionar futuros estudos nesse cenário.

\section{REFERÊNCIAS}

1. Kumakech E, Achora S, Berggren V, Bajunirwe F. Occupational exposure to HIV: a conflict situation for health workers. Int Nurs Rev. [Internet] 2011; 58(4) [acesso em 08 dez 2015]. Disponível:

http://dx.doi.org/10.1111/j.1466-7657.2011.00887.x.

2. Joyce MP, Kuhar D, Brooks JT. Notes from the field: occupationally acquired HIV infection among health care workers - United States, 1985-2013. MMWR Morb Mortal Wkly Rep. [Internet] 2015; 63(53) [acesso em 24 mar 2016]. Disponível: https://www.cdc.gov/mmwr/preview/mmwrhtml/mm6353a4.htm.

3. Kuhar DT, Henderson DK, Struble KA, Heneine W, Thomas V, Cheever LW, et al. Update U. S. public health service guidelines for the management of occupational exposures to HIV and recommendations for postexposure prophylaxis. Infect Control Hosp Epidemiol. [Internet] 2013; 34(9) [acesso em 9 mai 2016]. Disponível:

http://www.jstor.org/stable/10.1086/672271.

4. Brasil. Ministério do Trabalho e do Emprego. Portaria n. 485, de 11 de novembro de 2005. Aprova a Norma Regulamentadora $n^{\circ} 32$ (Segurança e Saúde no Trabalho em Estabelecimentos de Saúde). Diário Oficial da União, Brasília, DF, 16 nov. 2005, Seção 1.

5. Guilarde AO, de Oliveira AM, Tassara M, de Oliveira B, de Andrade SS. Acidentes com material biológico entre profissionais de Hospital Universitário de Goiânia. Rev. Patol. Trop. [Internet] 2010; 39(2) [acesso em 12 jul 2014]. Disponível: http://dx.doi.org/10.5216/rpt.v39i2.10730. 
6. Julio RS, Filardi MB, Marziale MHP. Acidentes de trabalho com material biológico ocorridos em municípios de Minas Gerais. Rev. bras. enferm. [Internet] 2014; 67(1) [acesso em 05 dez 2015]. Disponível:

http://dx.doi.org/10.5935/0034-7167.20140016.

7. Ministério da Saúde (BR). Gabinete do Ministro. Portaria n. 963, de 27 de maio de 2013. Redefine a Atenção Domiciliar no âmbito do Sistema Único de Saúde (SUS). Diário Oficial da União, DF, Brasília (DF): Ministério da Saúde; 2013. Seção 1.

8. Marques ALN, Ferreira MBG, Duarte JMG, Costa NS, Haas VJ, Simões ALA. Qualidade de vida e contexto de trabalho de profissionais de enfermagem da Estratégia Saúde da Família. Rev. Rene. [Internet] 2015; 16(5) [acesso em 05 abr 2016]. Disponível: http://www.revistarene.ufc.br/revista/index.php/revista/article/view/2058/pdf.

9. Conselho Federal de Enfermagem (COFEN). Perfil dos profissionais de enfermagem. [Internet] Brasília (DF): COFEN; 2015 [acesso em 08 jan 2016]. Disponível:

http://www.cofen.gov.br/pesquisa-inedita-traca-perfil-da-enfermagem_31258.html.

10. Facchin LT, Gir E, Pazin-Filho A, Hayashida M, Canini SRS. Under-reporting of accidents involving biological material by nursing professionals at a Brazilian emergency hospital. Int. J. Occup. Saf. Ergon. [Internet] 2013; 19(4) [acesso em 08 nov 2015]. Disponível: http://dx.doi.org/10.1080/10803548.2013.11077011.

11. Pires D, Lopes MGD, da Silva MCN, Lorenzetti J, Peruzzo SA, Bresciani HR. Jornada de 30 horas semanais: condição necessária para assistência de enfermagem segura e de qualidade. Enferm. Foco. [Internet] 2010; 1(3) [acesso em 03 abr 2016]. Disponível: http://revista.portalcofen.gov.br/index.php/enfermagem/article/view/182.

12. Wicker S, Stirn AV, Rabenau HF, von Gierke L, Wutzler S, Stephan C. Needlestick injuries: causes, preventability and psychological impact. Infection. [Internet] 2014; 42(3) [acesso em 24 mai 2016]. Disponível:

http://dx.doi.org/10.1007/s15010-014-0598-0.

13. Garbaccio JL, Regis WCB, Silva RMC, Estevão WG. Acidentes ocupacionais com a equipe de enfermagem da atenção hospitalar. Cogitare Enferm. [Internet] 2015; 20(1) [acesso em 04 abr 2016]. Disponível:

http://dx.doi.org/10.5380/ce.v20i1.37661.

14. Ministério da Saúde (BR). Secretaria de Atenção à Saúde. Departamento de Atenção Básica. CoordenaçãoGeral de Atenção Domiciliar. Caderno de atenção domiciliar. Brasília: Ministério da Saúde; 2012.

15. de Figueiredo RM, Maroldi MAC. Internação domiciliar: risco de exposição biológica para a equipe de saúde. Rev. esc. enferm. USP. [Internet] 2012; 46(1) [acesso em 05 jul 2014]. Disponível:

http://dx.doi.org/10.1590/S0080-62342012000100020.

16. Rezende KCAD, Tipple AFV, Siqueira KM, Alves SB, Salgado TA, Pereira MS. Adesão à higienização das mãos e ao uso de equipamentos de proteção pessoal por profissionais de enfermagem na atenção básica em saúde. Cienc. Cuid. Saude. [Internet] 2012; 11(2) [acesso em 06 abr 2016] Disponível:

http://dx.doi.org/10.4025/cienccuidsaude.v11i2.15204.

17. Gallas SR, Fontana RT. Biossegurança e a enfermagem nos cuidados clínicos: contribuições para a saúde do trabalhador. Rev. bras. enferm. [Internet] 2010; 63(5) [acesso em 10 fev 2015]. Disponível:

http://dx.doi.org/10.1590/S0034-71672010000500015.

18. Neves HCC, Souza ACS, Medeiros M, Munaris DB, Ribeiros LCM, Tipple AFV. Segurança dos trabalhadores de enfermagem e fatores determinantes para adesão aos equipamentos de proteção individual. Rev. Latino-Am. Enfermagem. [Internet] 2011; 19(2) [acesso em 4 abr 2016]. Disponível:

http://dx.doi.org/10.1590/S0104-11692011000200018.

19. Leiss JK. Provision and use of safety-engineered medical devices among home care and hospice nurses in North Carolina. Am J Infect Control. [Internet] 2010; 38(8) [acesso em 12set2015]. Disponível:

http://dx.doi.org/10.1016/j.ajic.2010.01.017.

20. Xi H, Cao J, Liu J, Li Z, Kong X, Wang Y, et al. Improving health care workers' protection against infection of Ebola hemorrhagic fever through video surveillance. Am J Infect Control. [Internet] 2016; 44(8) [acesso em 25 mai 2016]. Disponível: http://dx.doi.org/10.1016/j.ajic.2016.02.015. 